\title{
Computer-mediated communication in DIY discourse
}

\author{
Svetlana Pervukhina ${ }^{*}$, and Mikhail Churikov ${ }^{2}$ \\ ${ }^{1}$ Don State Technical University, 1 Gagarina Sq., Department "World Languages and Cultures", \\ 344000, Russia \\ ${ }^{2}$ Rostov State Transport University, 2, Rostovskogo Strelkovogo Polka Narodnogo Opolcheniya Sq., \\ 344038 Rostov-on-Don, Russia
}

\begin{abstract}
Do-It-Yourself discourse (DIY discourse) is communication aimed at teaching laymen to do professional processes and exchanging experiences in professional spheres. It is a very called-for tool for nonvocational training. DIY discourse comprises non-verbal, verbal, and polycode texts. In this research we analyzed general characteristics of DIY discourse. On the first stage of our research we selected articles from www.wikihow.com that teach the lay audience how to do different things. On the second stage of the research we analyzed genres that occur in the computer-mediated environment and polycode texts that represent the computer-mediated communication (CMC) in the DIY discourse. From a semiotic point of view, non-verbal texts are an emblem of a professional process, verbal texts provide a symbol side, and polycode texts combine an emblem and a symbol. We have found out that DIY discourse comprises several genres with different semiotic codes. It can have non-verbal, verbal and polycode format. We have come to the conclusion that polycode texts are the specific feature of computer-mediated communication. They are widely employed in DIY discourse to achieve a more profound comprehension of the professional processes.
\end{abstract}

\section{Introduction}

A lot of researches are interested in different discourses [1,2], and many of them are connected with computer technologies. Digital communication has changed a lot of spheres, and professional and educational discourses as well $[3,4]$. Computer-mediated communication (CMC) became a widely-used term in linguistics [5] because it is a very useful tool for mass communication, a necessary medium for global communication of likeminded people. Do-It-Yourself science combines features of professional discourse, educational discourse, and science, attracting a lot of people both professionals and laymen who are eager to exchange information and experience with each other [6]. Do-It-Yourself discourse (DIY discourse) is a kind of discourse that happens among people who share same interests in Do-It-Yourself (DIY) and exchange their knowledge and experience. This sphere is of interest for linguists for a number of reasons. First of all, it is a sphere of CMC

\footnotetext{
*Corresponding author: s pervuhina@mail.ru
} 
that has its own special peculiar features in comparison with non-mediated communication. Secondly, DIY discourse reflects exchanging information about technological processes that have been invented or explored and do not have any specific terms yet. So, ways of nominating processes is a very important issue for semiotics because in CMC the nominating technological processes may have various forms, both verbal and non-verbal; nominations in this discourse can be given as an oral speech or written speech, as it has already been studied for news discourse [7]. The aim of this article is to describe characteristic features of computer-mediated communication in DIY discourse.

\section{Method and materials}

The globalization provides opportunities for collaboration between a wide variety of people who would never have started communicating without digital technology and CMC. This feature of the World Wide Web is currently actively used in professional communities: the exchange of professional knowledge and experience has become more accessible and international. It is widely exploited by different professional communities that have their sites on Facebook or have their own domains as DiscourseNet (on discourseanalysis.net).

On the first stage of our research let us describe the DIY discourse.

On the second stage of our research we will provide specific details of the computermediated environment as a condition of contemporary DIY discourse.

On the third stage we will scrutinize specific genres of DIY discourse in CMC. This discourse uses creolized texts, that is a text with verbal and non-verbal components, and video articles as a specific polycode text with oral-written verbal and non-verbal components. We discuss a polycode text with its hybrid nature as a new type of computermediated communication, which has appeared due to the computer literacy of participants of communication.

On the fourth stage of our research we will detail the communicative aim of DIY discourse and peculiarities of its participants.

\section{Results}

\subsection{General characteristics of DIY discourse}

DIY is a very popular trend among people who are fond of handmade furniture, mending techniques, decorating houses themselves, and so on. These things can be done by professionals, but it either costs a lot, so some people prefer to do it themselves; or it is pleasant for them to do something in their own way. Surely, it is a sphere where we can find amateurs (or laymen). Mostly, they communicate informally, and computer technologies broadened the boundaries of such communication. It has become international, and it has acquired a new type of texts - polycode texts, which will be discussed further in detail.

DIY becomes more and more popular, thanks to CMC a lot of information is available for those who want to get new knowledge and acquire new skills. In professional training preciseness of the descriptions is of a very high value. While reading the text, the addressee should not have any doubts about repetition of the process. Everything should be perfectly clear. It determines the appearance of new genres with alternative semiotic means of transferring information.

DIY is an umbrella term that has a lot of synonyms, such as citizen science, open science, civic science, etc. This trend developed into DIY labs that offer users physical and social spaces [8]. According to N.D. Arutyunova, the discourses can be divided into person- 
oriented and status-oriented [9]. Institutional discourses have an organizational structure with hierarchy where communication takes place; the addressor and the addressee have certain roles there. Non-institutional discourse does not have any organization where it can happen, and it does not have any hierarchy among the communicants, either [9].

DIY discourse is a person-oriented non-institutional discourse. Its main idea is to describe the process of producing things and the final product of this process. The preciseness of description and readability of the text are of high importance for this type of discourse. That is why the text might include both verbal and non-verbal components. DIY discourse comprises non-verbal, verbal, and polycode texts. From a semiotic point of view, non-verbal texts are an emblem of a professional process. It means that they mirror a part of the first signal system that includes this process, and the addressee can see this process in the reflection (picture) that accompanies a verbal message.

Verbal texts provide a symbol side because they use the second signal system - words of the natural language - that also describes the process of DIY, but nevertheless provides some extra information. It overlaps the non-verbal information that might be necessary or interesting for the addressee.

Polycode texts combine an emblem and a symbol. On Fig.1 you can see pictures reflecting the first signal system (the outer world), and the process of planting a rose can be seen in the stages required for planting accompanied by short instructions summing up what is presented in the picture.

As a rule, DIY discourse happens among the lay audience. But for the reason of acquiring true knowledge and correct skills, the consultation of a specialist in that sphere is needed. On the resource that we have analyzed there are two ways of specialist consultation - oral and written. An oral form of the consultation is a video episode with an interview where you can see all the process from the beginning till the end, and hear about its details.

So, the DIY texts are hybrid texts that can be referred both to oral and written communication at the same time (in case they have the video episodes with interviews), they have both verbal and non-verbal methods of representing the information. So, it is a completely new way of transmitting the information in a professional sphere that has appeared only due to CMC. Consultations or interviews with the specialist has always been a part of the professional discourse (or a DIY discourse), but they started to be combined with verbal texts in CMC.

While describing things, the addressor tries to detail the process, describe the materials needed, highlight the details that might slip the attention of the addressee, but which are very important for achieving the result. On Fig.1 the process of replanting a rose bush is depicted (according to a DIY resource www.wikihow.com).

Steps 1 and 5 are pictures without any signal signs.

Step 1 shows a place where a rose bush can be planted, Step 5 indicates the stage of digging the rose bush out.

Steps 2,3,4 and 6 (Fig.1) have got arrows and dotted lines to focus the reader's attention on certain elements of the process.

Step 2 provides an iconic sign of a water drop to indicate the humidity of the soil.

Step 3 illustrates the dimensions of a hole for a rose bush. Step 6 shows the level of soil filling, and the arrow indicates the direction of throwing the soil.

Step 4 has got a dotted line to draw the reader's attention to the important detail of the picture.

On Fig. 1 a lot of small details are explicated in either verbal or non-verbal form. These forms supplement each other. And both of them have extra information.

Fig.1 illustrates creolized texts of DIY discourse, each picture showing a stage of the process. Important details of the process are marked by words, denoting necessary 
dimensions, distances, etc. All these DIY figures are followed by a detailed verbal instruction.
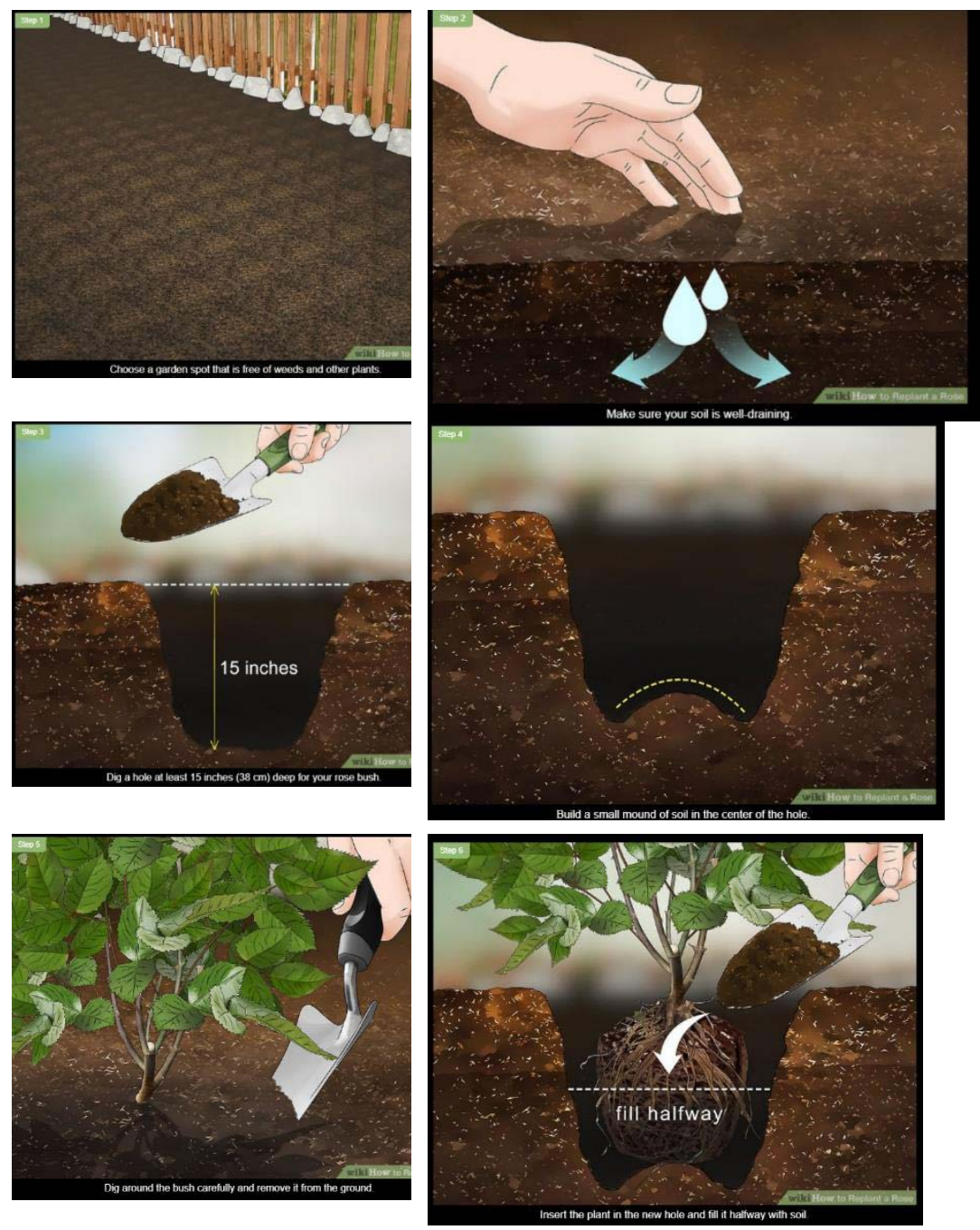

Fig. 1. Pictures denoting steps of a process in DIY discourse, Source https://www.wikihow.com/Replant-a-Rose

For example, Step 3 is described as follows (Fig.2):

3 Dig a hole at least 15 inches $(38 \mathrm{~cm})$ deep for your rose bush. Use a garden

trowel to dig out a new spot for your rose bush. Prepare a hole that is about 15 inches $(38 \mathrm{~cm}$ ) deep. The hole should also be at least 12 inches $(30 \mathrm{~cm})$ wide, or large enough to accommodate your rose bush. ${ }^{[5]}$

- Wear gardening gloves to protect your hands as you dig.

Fig. 2. Description in a creolized text. Source https://www.wikihow.com/Replant-a-Rose 
The picture (Fig.1, step 3) and the description below (Fig.2) comprise a creolized text where non-verbal and verbal parts are connected with each other. The verbal part duplicates the information from the picture about the tool to be used, the dimensions of the hole for planting, etc.; it also gives a proviso about a possible discrepancy in size. We can find extra verbal information with warning about protecting the hands. Actually, all steps of the instruction followed the same pattern: the verbal components provide duplicating information about the process and some extra information about it. Nevertheless, the picture gives an emblematic sign of the process, which refers to the first signal system and corresponds to the personal experience.

DIY discourse can be a useful ground for non-formal learning that is really called-for in Russia now. As E.Murugova and T.Mikheeva state, it is a very challenging but highly recommended trend [10].

\subsection{Computer-mediated environment}

The dynamic environment of communication invariably gives rise to new genres, since the Internet with its technical innovations is the trigger for their creation. Modern children learn to use technologies from early childhood for it becomes a part of a communication habit. A. Kesharwani introduces a term 'digital natives' that stands for children who were born in the world with technologies [11]. But adults learn them too, and become 'digital immigrants', as A. Kesharwani states it [11]. So, at the moment, the world is experiencing two different modes of communication. The first one presupposes verbal texts (oral or written) that can be long enough and from a semiotic point of view bear a symbol nature. The signs of the symbolic system do not have any resemblance either with the objects or with the notions that they denote. The second one, CMC, presupposes an iconic nature that consists in reflecting the objects of the surrounding world. The advantage of CMC is in the technologies that can reflect the processes described and transmit oral speech when it is necessary. In this respect, $\mathrm{CMC}$ does have the verbal component, but the message is visualized greatly. We will speak about it in more detail in 3.2.1.

The ability of a person to adapt to the changing conditions of communication is manifested in the invention of new genres that meet the needs of communication to the highest degree $[12,13,14]$. Polycode texts have been attracting the interest of researchers for a long time because they give new opportunities to express complicated ideas from the point of view of cognitive logical information [15,16], and from the point of view of connotative information in advertising texts [17]. So, the nonverbal component of such texts serves for the following purposes in the DIY discourse:

(1) it gives a visual nomination of a process, so that the reader sees the process and is able to imitate it. Meanwhile, the verbal description of such a process may lead to misunderstanding and consequently to misperformance due to approximation of nominations of the addressor and the addressee;

(2) pictures and visuals draw attention of the reader, bright colours create a certain emotional state, so the non-verbal component transfers connotative information.

Close attention was paid to advertising texts, as well as semiotic and semantic aspects of the components of the creolized text. There is an extensive bibliography of studies that investigate the textual component of films in cinematic discourse $[18,19,20$, etc.]. However, polycode texts with a video component, functioning in professional communication, have escaped the researcher's attention. 


\subsubsection{Polycode texts}

Visualization is a very important issue in DIY communication, especially if the matter is concerned learning new skills [21]. There is a terminological problem in the difference between the terms "creolized text" and "polycode text". The term "creolized" was introduced to emphasize the hybrid properties of this object, but many authors [22,23, etc.] prefer the term "polycode", i.e. created on the basis of different semiotic systems for the transferring of a single message. In our research we distinguish these terms. Creolized texts belong to written communication, they combine two elements. - verbal and non-verbal. The prefix 'poly-' is good for determining multiple elements of hybrid nature.

In the case of video articles in DIY discourse, the latter term seems to be more successful, since it emphasizes several components of such a text: 1) a written text component, 2) a video-audio component in the form of an attached video file as a part of the article, 3) an emblemic component in the form of pictures and photographs.

Thus, a polycode text is understood as an inhomogeneous text that conveys information through several codes:

1) written-verbal;

2) oral-verbal;

3) non-verbal (iconic), presented in the form of images and videos.

Fig. 1 and Fig. 2 represent written-verbal and non-verbal codes describing one and the same message. In 3.2.2 we will discuss the texts with all three codes.

In fact, practically all standard instructions are creolized texts because they have figures with illustrations and captures with extra details. Polycode texts can be created only with the help of technologies, and they are readily created by digital natives who are able to combine different codes (sounds, images, texts, and videos) in their messages; such texts can perform different functions and can be found in a number of discourses (e.g. postcards and congratulations via messenger programs, blogs, stories in Instagram are all different genres in CMC can be polycode texts).

For DIY discourse is a discourse of communication with exchanging specific experience, a polycode nature of such a text ensures more comprehensible format for a reader. A hybrid format represents a new level of text perception that is performed on the abstract level of language and on the subject level of senses (seeing and hearing).

\subsubsection{Video articles as a genre of DIY discourse}

It should be highlighted that a video article occupies an intermediate position between oral and written communication because of its hybrid form, since it combines oral and written communication in one message. The importance of the genre of video articles is determined by the digital environment of modern communication and is limited by the technological devices and the user's skills.

Video articles of a DIY discourse became the material of our research because, firstly, they represent a new genre of CMC and, secondly, they present a new mode of transferring information using new technologies.

A usual composition of a video article is as follows:

- $\quad$ an introduction stating the importance of the question;

- creolized texts denoting stages of a process. They consist of an image (a non-verbal component) accompanied by a verbal explanation. It overlaps the information in the picture and provides new facts and details;

- commentaries with clarifying questions about the process in the form of a forum. They are answered by experts in this field; 
- a video with explanation of extra details and presentation of a definite device or a process;

- $\quad$ links to related articles;

- contents.

These stages demonstrate a multifaceted vision of a fragment of the world - a process or a subject. The introduction gives a general idea of the issue. The function of this stage is similar to a usual introduction to an article. The creolized texts demonstrating the steps of the process give a schematic view of the denoted process. At this stage there can be questions from the lay audience who might miss something from the description or the explanation. So, the readers may ask a question and get a feedback. The following step is a video part of the article where a reader/viewer can see the process performed by a specialist and can listen to an explanation of what is happening in the episode. Then there is a step that provides links to the articles or resources connected with the topic described. Thus, a video article provides both generalized and specific information about the topic, it presents views of people with different levels of proficiency in the topic. The last step is contents that have hyperlinks to all parts of the article.

A video article can give safety instructions, according to https://www.wikihow.com/Use-a-Weed-Whacker. On the one hand, it describes a process of weed whacking that can be risky if safety instructions are not observed. On the other hand, it demonstrates a definite device and displays how to use it. Moreover, the presenter speaks about the detail (oral speech) and shows this device and how it works (non-verbal cinema part). This video article also provides generalized images of a typical weed whacker and how to use it safely (creolized texts with written verbal and non-verbal components).

It should be noted that a video article is interactive in its structure, which means that in some parts it has a dialogue traits. It also has got features of a hypertext (links to other resources).

The topic of a video article can be a device as in the previous example or the process as in https://www.wikihow.com/Write-a-Standard-Operating-Procedure. This article is about writing a document, so the denotate is rather abstract.

The video is a transition to the first signaling system, which allows you to show what is difficult to explain in words in any language. There are some aspects of the professional experience that are more efficiently conveyed through non-verbal channels. This experience is associated with the accuracy of actions, the manipulation of mechanisms, the operation of machines and devices necessary for professional activity. At the same time, verbal commenting on actions, description of an extra-textual situation is important for the exchange of professional knowledge base.

In some cases (for example, in https://www.wikihow.com/Make-Tempura) the creolized texts have videos as parts of the descriptive stage. They may substitute the expert video stage. The verbal part of the article presents an instruction that is short and precise. The video duplicates the subjects (nouns) and the action in the instruction. For example, 'ice water' is shown as water with cubes of ice. In this example, the verbal part is not a complete repetition of the action in the film. It has some extra information (dimensions, nominations, details of the process).

Thus, the analysis of a video article as a genre within the framework of DIY discourse is promising from the point of view of tracing the ways of nominating objects, processes and notions. 


\subsubsection{DIY commentaries and their pragmatic function}

Analyzed DIY video articles have got commentary parts. It represents questions from the lay audience who is interested in the issue and the answers of an expert. In fact, in this way a reader may get a feedback from an expert. It is a certain comprehension check. In this way, a video article is totally different from a written instruction or a journal article, for instance. The experts in their turn, also have a feedback from the readers. They mark if the answer is useful or not, interesting or not. The system of 'likes' is a specific feature of CMC. It marks the popularity of the article or its writer.

Usually, such commentaries are moderated, and the most relevant ones are allowed in the site. We do not consider it is as a distortion of the polycode text, for texts in the written communication can be checked and corrected. In fact, this feature is a characteristic for polycode texts that occupy an intermediary position between written and oral communication.

\subsubsection{DIY verbal texts and the professional commentary}

The interaction between the reader and the writer is very important in this genre. The writer is seen as an expert (though in some cases it can be an experienced amateur). The advice of such a person (professional and experienced) is very important for the reader, so the professional opinion is an integrative part of an article. It can be given in the form of a video (in the oral and visual form) or it can be given as a written text, as in https://www.wikihow.com/Help-a-Narcissist.

\subsection{Communicative aim and participants in DIY discourse}

Experts, whose experience is very valuable for the professional community, or amateurs with some know-how, share their knowledge about DIY with people who are interested in their skills through video clips. Such stories are valuable for those who want to improve in the professional field, especially since the video can be accompanied by verbal explanations. A polycode speech message consisting of a verbal component that is logically and structurally related to the iconic component, which is a video fragment, is called a video article. Video article as a speech genre has not been previously considered by linguists, although this genre has been functioning for a long time. The analyzed material allowed us to draw some conclusions about CMC and DIY discourse.

On the one hand, a new mode of communication, $\mathrm{CMC}$, is very demonstrative. It helps to acquire certain skills with the help of instructions. On the other hand, it has got its limitations. Mostly, they are connected with the focus of reader's attention on this or that detail of the picture.

Readers, or addressees, can have a many faceted view of the situation in the videoarticle due to its diversity in semiotic systems that are used to describe professional processes.

\section{Discussion}

Our research has been devoted to a new type of texts - polycode texts - which can be found in DIY discourse. Investigation of such texts is really important for linguistics now because we see a drastic change in the whole system of communication with computer technologies. Looking back into the ages we can see three types of texts. In oral culture with no written language, the texts were to be memorized. So, we can suppose that there were not a big amount of culturally or vitally important texts that were transmitted from generation to generation orally and they had to be memorized to exist. After appearance of a written 
language and a printing press, the situation changed. The texts could be stored, and they could exist independently from someone's memory; moreover, they could be multiplied. Now we are witnessing a new type of communication which is a new type of culture, that is a computer-mediated culture. The texts have to be neither memorized nor printed to exist. They can be multiplied in a greater number of copies, and the speed of dissemination is really high. Thus, polycode texts are a civilization leap that has changed communication and is transforming some discourses. DIY is one of the examples of such transformation. Our analyzed material showed that lay audience can no longer need a professional who would explain things in person. Online communication has changed the situation, and such sites as https://www.wikihow.com/ can provide people with all necessary visual and verbal information, can ensure a proper feedback.

One more aspect that is worth studying in different types of discourses and cultures is addressor's status. In oral cultures the addressor (or a story-teller) had a very high status for he or she was the keeper of the unique knowledge. It was their memory that was the condition of the knowledge existence. In written cultures this status was transferred to the libraries. There were a lot of books there, and they were available for public. In CMC, the greatest storage of information is certainly the Internet, and the status is transferred from the addressor to the addressee with proper computer skills. M. Nguyen et al. explored different status of computer users caused by their computer skills and availability of devices during the Covid pandemic [24]. They found out that there was a strict dependence of status and career prospects. Their findings have been proved by A. Reznikova et al. [25]. All in all, the researchers claim the paramount importance of the computer skills nowadays and their extreme usefulness.

As A.Reznikova et al. noted, modern communication systems state an alternative for teaching due to their ability to use hybrid polycode texts [25], with written-oral verbal and non-verbal mode of communication. Actually, it is possible to consider 'a recorded mode' that has already been implemented in researches of media discourse as opposite to 'on air'.

In conclusion it is necessary to mark the importance of $\mathrm{CMC}$ investigation for linguistics and other humanitarian disciplines.

\section{References}

1. E. Murugova, N. Boeva-Omelechko, M. Zheltukhina, O. Ryabko, G. Matveeva, I. Zyubina. Space and Culture, India, 6 (4), 112-121 (2018) doi.org/10.20896/saci.v6i4.387

2. E. Sakharova, N. Revyakina, E3S Web of Conferences 210, 18040 (2020) doi.org/10.1051/e3sconf/202021018040

3. S.Pervukhina, G.Matveeva. WUT-2020, 1064-1074 (2020) doi.org/10.15405/epsbs.2020.08.124

4. S.Pervukhina, G.Radchenko. E3S Web of Conferences 210, 1-10, 18036 (2020) doi.org/10.1051/e3sconf/202021018036

5. A.Lyons, Journal of Pragmatics, 131, 18-29 (2018) doi:10.1016/j.pragma.2018.05.001

6. D. Sarpong, G.Ofosu, D.Botchie, F. Clear. Technological Forecasting and Social Change, 158, 120127 (2020). doi:10.1016/j.techfore.2020.120127

7. R. Facchinetti, Journal of Pragmatics, 175, 195-206 (2021) doi.org/10.1016/j.pragma.2021.01.015

8. Y. Weimu, C. Weifeng, M. Agyapong, C. Mordi, Technological Forecasting and Social Change 159, 120205 (2020) 
9. V.V. Karasik, D. Gillespie, Procedia - Social and Behavioral Sciences 154, 23 29 (2014) https://doi.org/10.1016/j.sbspro.2014.10.106

10. E.V. Murugova, T. B. Mikheeva. E3S Web of Conferences, 210, 18095 (2020) doi: doi.org/10.1051/e3sconf/202021018095

11. A.Kesharwani, Information \& Management, 57, 103170 (2019) doi:10.1016/j.im.2019.103170

12. A.Sampietro, Journal of Pragmatics, 143, 109-120 (2019) https://doi.org/10.1016/j.pragma.2019.02.009

13. M. Kuteeva, A.Mauranen, Discourse, Context \& Media, 24, 1-7 (2018) doi:10.1016/j.dcm.2018.06.001

14. C. D. Maier, S. Ravazzani, International Journal of Business Communication, 232948841876869 (2018). doi:10.1177/2329488418768690

15. M.Mailer, Organizational psycholinguistics, 1 (9), 1-12 (2020)

16. S.V. Dmitrichenkova, \& E.A. Dolzhich, Procedia Computer Science, 103, 584588 (2017) doi:10.1016/j.procs.2017.01.071

17. E. Anisimova, Advertising communication: linguistic and cultural aspect, 124-133 (2011)

18. M.A. Samkova, Philological sciences. Questions of theory and practice 1 (8), 135 137 (2011)

19. G.G. Slyshkin, M.A. Efremova. Kinotext (experience of cultural analysis) (M .: Vodoley, 2004)

20. J.A. Bateman, L.Thiele, H.Akin, Journal of Pragmatics, 175, 112-128 (2021) https://doi.org/10.1016/j.pragma.2020.12.009

21. G.N. Ostrikova, M.R. Zheltukhina, I.A. Zyubina, I.G. Sidorova, Astra Salvensis. 6(1), 601-607 (2018)

22. E.V. Chernyavskaya, Linguistics of the text. Polycode, intertextuality, interdiscursiveness (2009)

23. S.V. Dmitrichenkova, E.A. Dolzhich, Procedia Computer Science, 103, 584-588 (2017) doi:10.1016/j.procs.2017.01.071

24. M.H. Nguyen, E. Hargittai, W.Marler, Computers in Human Behavior, 120, 106717 (2021). https://doi.org/10.1016/j.chb.2021.106717.

25. A. Reznikova, T. Kudinova, R. Patuykova, N. Olomskaya, O.Dyshekova, E3S Web of Conferences, 210, 18037 (2020) https://doi.org/10.1051/e3sconf/202021018037 